\title{
Origin of the Ising Ferrimagnetism and Spin-Charge Coupling in $\mathrm{LuFe}_{2} \mathrm{O}_{4}$
}

\author{
H. J. Xiang, ${ }^{1}$ E. J. Kan, ${ }^{2}$ Su-Huai Wei, ${ }^{1}$ M.-H. Whangbo, ${ }^{2}$ and Jinlong Yang ${ }^{3}$ \\ ${ }^{1}$ National Renewable Energy Laboratory, Golden, Colorado 80401, USA \\ ${ }^{2}$ Department of Chemistry, North Carolina State University, \\ Raleigh, North Carolina 27695-8204, USA \\ ${ }^{3}$ Hefei National Laboratory for Physical Sciences at Microscale, \\ University of Science and Technology of China, Hefei, Anhui 230026, P. R. China
}

(Dated: October 8, 2018)

\begin{abstract}
The spin ordering and spin-charge coupling in $\mathrm{LuFe}_{2} \mathrm{O}_{4}$ were investigated on the basis of density functional calculations and Monte Carlo simulations. The 2:1 ferrimagnetism arises from the strong antiferromagnetic intra-sheet $\mathrm{Fe}^{3+}-\mathrm{Fe}^{3+}$ and $\mathrm{Fe}^{3+}-\mathrm{Fe}^{2+}$ as well as some substantial antiferromagnetic $\mathrm{Fe}^{2+}-\mathrm{Fe}^{3+}$ inter-sheet spin exchange interactions. The giant magnetocapacitance at room temperature and the enhanced electric polarization at $240 \mathrm{~K}$ of $\mathrm{LuFe}_{2} \mathrm{O}_{4}$ are explained by the strong spin-charge coupling.
\end{abstract}

PACS numbers: 75.80.+q,71.20.-b,77.80.-e,64.60.De 
Recently, multiferroics [1, 2, 3, 4, 5, 6, 7, 8, 9, 10] have attracted much attention because of their potential applications in novel magnetoelectric and magneto-optical devices. Among the newly discovered multiferroics, $\mathrm{LuFe}_{2} \mathrm{O}_{4}$ is particularly interesting due to its large ferroelectric (FE) polarization [3] and giant magnetocapacitance at room temperature [4]. In the high-temperature crystal structure of $\mathrm{LuFe}_{2} \mathrm{O}_{4}$ with space group $\mathrm{R} \overline{3} \mathrm{~m}$, layers of composition $\mathrm{Fe}_{2} \mathrm{O}_{4}$ alternate with layers of $\mathrm{Lu}^{3+}$ ions, such that there are three $\mathrm{Fe}_{2} \mathrm{O}_{4}$ layers per unit cell. Each $\mathrm{Fe}_{2} \mathrm{O}_{4}$ layer is made up of two triangular sheets (hereafter, T-sheets) of corner-sharing $\mathrm{FeO}_{5}$ trigonal bipyramids (Fig. 1). Below $320 \mathrm{~K}\left(T_{C O}\right) \mathrm{LuFe}_{2} \mathrm{O}_{4}$ undergoes a three-dimensional (3D) charge ordering (CO) $\left(2 \mathrm{Fe}^{2.5+} \Rightarrow \mathrm{Fe}^{2+}+\mathrm{Fe}^{3+}\right)$ with the $\sqrt{3} \times \sqrt{3}$ superstructure in each T-sheet; in each $\mathrm{Fe}_{2} \mathrm{O}_{4}$ layer, one T-sheet has the honeycomb network of $\mathrm{Fe}^{2+}$ ions with a $\mathrm{Fe}^{3+}$ ion at the center of each $\mathrm{Fe}^{2+}$ hexagon (hereafter, the type A T-sheet), while the other T-sheet has an opposite arrangement of the $\mathrm{Fe}^{2+}$ and $\mathrm{Fe}^{3+}$ ions (hereafter the type B T-sheet).

$\mathrm{LuFe}_{2} \mathrm{O}_{4}$, with the novel CO-driven "electronic ferroelectricity", [3] presents several fundamental questions. First, $\mathrm{LuFe}_{2} \mathrm{O}_{4}$ shows strong Ising behavior with the easy axis along $c$ [11, 12]. The spin anisotropy of the non-CO state is understandable because the spin down electron of the $\mathrm{Fe}^{2.5+}$ ion partially occupies the degenerate $\left(d_{x^{2}-y^{2}}, d_{x y}\right)$ orbitals [5, 13]. However, the Ising behavior below $T_{C O}$ is puzzling because the insulating $\sqrt{3} \times \sqrt{3}$ CO breaks the 3 -fold rotational symmetry hence lifting the degeneracy of the $\left(d_{x^{2}-y^{2}}, d_{x u}\right)$ orbitals $[5]$. Second, $\mathrm{LuFe}_{2} \mathrm{O}_{4}$ undergoes a ferrimagnetic spin ordering below $240 \mathrm{~K}\left(T_{N}\right)$ [11, 14, 15, 16]. A number of experimental studies found this spin ordering to be two-dimensional (2D) in nature [11, 14, 17]. In contrast, a recent neutron diffraction study observed a finite spin correlation along $c$ and suggested a 3D spin structure without considering CO [16]. The Mössbauer [14] and neutron diffraction [15] studies led to a detailed ferrimagnetic structure of $\mathrm{LuFe}_{2} \mathrm{O}_{4}$, in which the majority spin lattice consists of all $\mathrm{Fe}^{2+}$ ions plus one-third of the total $\mathrm{Fe}^{3+}$ ions while the minority spin sublattice consists of the remaining $\mathrm{Fe}^{3+}$ ions. This 2:1 ferrimagnetic order was suggested to originate from weak ferromagnetic (FM) interactions between the next-nearest neighbor (NNN) Fe sites in the triangular antiferromagnetic (AFM) Ising lattice [11]. However, using the spin exchange parameters estimated from the energy parameters of $\mathrm{LaFeO}_{3}$, Naka et al. [18] predicted quite a different spin structure that includes some Fe sites without unique spin direction. Therefore, the detailed ferrimagnetic structure and its origin remain unclear. Third, $\mathrm{LuFe}_{2} \mathrm{O}_{4}$ exhibits a giant magnetodielec- 
tric response at room temperature [4], and a room-temperature dynamic magnetoelectric coupling was also reported [19]. Furthermore, the FE polarization of $\mathrm{LuFe}_{2} \mathrm{O}_{4}$ was found to increase around $T_{N}$ [3]. These observations suggest the occurrence of coupling between the $\mathrm{CO}$ and magnetism. The understanding of the spin-charge coupling is crucial for future magnetodielectric applications of $\mathrm{LuFe}_{2} \mathrm{O}_{4}$.

In this Letter, we explore these isuues on the basis of first principles density functional calculations for the first time. A large spin anisotropy is found along the $c$ direction due mainly to the $\mathrm{Fe}^{2+}$ ions of the B-sheet, the spin ground state of the $\sqrt{3} \times \sqrt{3} \mathrm{CO}$ state has the 2:1 ferrimagnetic spin arrangement proposed by Siratori et al. [15], and there occurs strong spin-charge coupling in $\mathrm{LuFe}_{2} \mathrm{O}_{4}$.

Our density functional theory calculations employed the frozen-core projector augmented wave method [20] encoded in the Vienna ab initio simulation package [21], and the generalized-gradient approximation (GGA) [22]. To properly describe the strong electron correlation in the $3 \mathrm{~d}$ transition-metal oxide, the GGA plus on-site repulsion $\mathrm{U}$ method $(\mathrm{GGA}+\mathrm{U})[23]$ was employed with the effective $U$ value $\left(U_{\text {eff }}=U-J\right.$ with $\left.J=0\right)$ of $4.61 \mathrm{eV}$ [5]. It is known experimentally [11, 14, 17] that the interlayer magnetic interactions in $\mathrm{LuFe}_{2} \mathrm{O}_{4}$ are weak, which is understandable due to its layered structure. In this work, therefore, we focus on the $2 \mathrm{D}$ spin ordering within a single $\mathrm{Fe}_{2} \mathrm{O}_{4}$ layer. For the $\sqrt{3} \times \sqrt{3}$ $\mathrm{CO}$ state of $\mathrm{LuFe}_{2} \mathrm{O}_{4}$, the $\mathrm{FE}$ ordering of the $\mathrm{Fe}_{2} \mathrm{O}_{4}$ layers will be assumed.

We first examine the magnetic anisotropy of the Fe ions by performing GGA+U calculations, with spin-orbit coupling (SOC) included, for the FM state of $\mathrm{LuFe}_{2} \mathrm{O}_{4}$ with the $\sqrt{3} \times \sqrt{3}$ CO. As shown in Fig. 1(a), there are two kinds of $\mathrm{Fe}^{2+}$ ions and two kinds of $\mathrm{Fe}^{3+}$ ions in the $\sqrt{3} \times \sqrt{3} \mathrm{CO}$ state. We label the $\mathrm{Fe}^{2+}$ and $\mathrm{Fe}^{3+}$ ions of the type A T-sheet as $2 \mathrm{~A}$ and $3 \mathrm{~A}$, respectively, and those of the type $\mathrm{B}$ T-sheet as $2 \mathrm{~B}$ and 3B, respectively. In our GGA+U+SOC calculations with spins pointing along several different directions, all $\mathrm{Fe}^{2+}$ and $\mathrm{Fe}^{3+}$ spins are kept in the same direction. Our calculations show that the easy axis is along the $c$ direction, as experimentally observed [11, 12]; the $\|$ c-spin orientation is more stable than the $\perp$ c-spin orientation by $1.5 \mathrm{meV}$ per formula unit (FU). The orbital moments of 2A, 2B, 3A, and 3B for the $\| \mathrm{c}$-spin orientation are 0.101, 0.156, 0.031 and 0.035, respectively, which are greater than those for the $\perp$ c-spin orientation by $0.019,0.062,0.015$, and $0.018 \mu_{B}$, respectively. As expected, the $\mathrm{Fe}^{3+}\left(d^{5}\right)$ ions have a very small anisotropy, However, two kinds of the $\mathrm{Fe}^{2+}$ ions also have different degree of spin anisotropy. The spin 
down electron of the $2 \mathrm{~B} \mathrm{Fe}{ }^{2+}$ ion occupies the $\left(d_{x^{2}-y^{2}}, d_{x y}\right)$ manifold [5], therefore the $2 \mathrm{~B} \mathrm{Fe}^{2+}$ ion has the largest spin anisotropy along $c$. Our calculations indicate a non-negligible orbital contribution to the total magnetization, in agreement with the X-ray magnetic circular dichroism result [12].

To determine the magnetic ground state of $\mathrm{LuFe}_{2} \mathrm{O}_{4}$ in the $\sqrt{3} \times \sqrt{3} \mathrm{CO}$ state, we extract its spin exchange parameters by mapping the energy differences between ordered spin states obtained from GGA+U calculations onto the corresponding energy differences obtained from the Ising Hamiltonian [24]:

$$
H=\sum_{i, j} J_{i j} S_{i z} S_{j z}
$$

where the energy is expressed with respect to the spin disorder (paramagnetic) state, $J_{i j}$ is the spin exchange parameter between the spin sites $i$ and $j$, and $S_{i z}$ is the spin component along the $c$ direction $\left(\left|S_{z}\right|=2\right.$ and 2.5 for $\mathrm{Fe}^{2+}$ and $\mathrm{Fe}^{3+}$ ions, respectively). We consider all 15 possible superexchange (SE) interactions and all 19 super-superexchange (SSE) interactions with the O...O distance less than $3.2 \AA$. The intra- and inter-sheet interactions within each $\mathrm{Fe}_{2} \mathrm{O}_{4}$ layer as well as the SSE interactions between adjacent $\mathrm{Fe}_{2} \mathrm{O}_{4}$ layers are taken into account. To evaluate these 34 spin exchange parameters reliably, we considered 111 different ordered spin states leading to 110 energy differences. The 34 spin exchange parameters were determined by performing a linear least-square fitting analysis. The SSE interactions are generally much weaker than the SE interactions with the magnitude of all SSE interactions less than $1.4 \mathrm{meV}$. The calculated SE parameters are reported in Table 【. All intra-sheet SE interactions are AFM, and the strongest interactions $(\sim 7.3 \mathrm{meV})$ occurs between the $3 \mathrm{~B} \mathrm{Fe}^{3+}$ ions because of the large energy gain of the AFM configuration and almost zero FM coupling. The inter-sheet SE interactions are weaker than the the intra-sheet SE interactions, and are mostly AFM.

With the calculated spin exchange parameters, one can identify the spin ground state of the CO state. The Metropolis Monte Carlo simulation of the Ising model is performed to search for the ground state. Simulations with supercells of several different sizes show that the spin ground state has the magnetic structure shown in Fig. 2(a), which has the same cell as the $\sqrt{3} \times \sqrt{3} \mathrm{CO}$ structure. In this state, all $\mathrm{Fe}^{2+}$ ions contribute to the majority spin, and the $\mathrm{Fe}^{3+}$ ions are antiferromagnetically coupled to the $\mathrm{Fe}^{2+}$ ions in the type A T-sheet. In the honeycomb lattice of the type B T-sheet, the $\mathrm{Fe}^{3+}$ spins are antiferromagnetically 
coupled. Thus, the spin ground state is ferrimagnetic, as experimentally observed [11]. This 2:1 ferrimagnetic structure is the same as the magnetic structure proposed by Siratori et al. [15], and differs from the structure proposed by Naka et al. [18].

The observed ferrimagnetic ordering can be readily explained in terms of the calculated exchange parameters. In the honeycomb network of the type B T-sheet, the nearest-neighbor (NN) 3B ions are antiferromagnetically coupled since their SE interaction is strongly AFM. In the type A T-sheet, the SE interactions between the 2A ions are AFM, and so are those between the $2 \mathrm{~A}$ and $3 \mathrm{~A}$ ions, which leads to spin frustration. As a consequence, two possible spin arrangements compete with each other in the type A T-sheet; the first is the state in which the coupling between the $\mathrm{NN} 2 \mathrm{~A}$ ions are AFM with the spin direction of the $3 \mathrm{~A}$ ion undetermined, and the second is the state in which all $2 \mathrm{~A}$ ions are antiferromagnetically coupled to the $3 \mathrm{~A}$ ions. The energies of these two states (considering only the SE interaction) are $E_{1}=-4\left(J_{2 A 1,2 A 2}+J_{2 A 1,2 A 4}\right)$ per $3 \mathrm{~A}$ ion, and $E_{2}=-10\left(J_{3 A 1,2 A 1}+J_{3 A 1,2 A 2}+J_{3 A 1,2 A 3}\right)+$ $4\left(J_{2 A 1,2 A 2}+J_{2 A 1,2 A 4}\right)$ per $3 \mathrm{~A}$ ion, respectively. Due to the relatively strong AFM interactions between the $3 \mathrm{~A}$ and $2 \mathrm{~A}$ ions (See Table I) and the large spin of the $3 \mathrm{~A}$ ions, the second state has a lower energy, i.e., $E_{2}<E_{1}$. Without loss of generality, we can assume the $2 \mathrm{~A}$ (3A) ions constitute the majority (minority) spin in the second state. Now, we examine the spin orientation of the $\mathrm{Fe}^{2+}$ ions in the type $\mathrm{B}$ T-sheet. The intra-sheet interactions of the $2 \mathrm{~B}$ ion with $3 \mathrm{~B}$ ions vanish due to the $\mathrm{AFM}$ ordering of the $3 \mathrm{~B}$ ions. As for the inter-sheet interactions involving the $2 \mathrm{~B}$ ions, the dominant one is the AFM interaction of the $2 \mathrm{~B}$ ion with the $3 \mathrm{~A}$ ion $\left(J_{3 A 1-2 B 1}\right.$ in Table $\left.\mathbb{I}\right)$. Consequently, we obtain the ferrimangetic ground state shown in Fig. 2(a), in which the spin of the $2 \mathrm{~B}$ ion contributes to the majority spin of the $\mathrm{Fe}_{2} \mathrm{O}_{4}$ layer. For the stability of the ferrimangetic ground state, the inter-sheet interaction is essential. This was neglected in the model Hamiltonian study of Naka et al. [18]. The ferrimangetic state is not due to the FM interactions between NNN Fe ions of the T-sheet because they must be vanishingly weak and mostly AFM.

The electronic structure of the ferrimangetic state calculated for the $\sqrt{3} \times \sqrt{3}$ CO structure of $\mathrm{LuFe}_{2} \mathrm{O}_{4}$ is shown in Fig. 3. Also shown is the electronic structure calculated for the FM state. Both states are semiconducting, and the highest occupied (HO) and the lowest unoccupied (LU) levels of both states come from the spin-up $\mathrm{Fe}^{2+}$ and $\mathrm{Fe}^{3+}$ ions, respectively [5]. In addition, the band dispersion from $\Gamma$ to $\mathrm{A}$ is rather small, indicating a very weak interlayer interaction. However, there are some important differences. First, the 
ferrimangetic state has a larger band gap $(1.68 \mathrm{eV})$ than does the FM state $(0.77 \mathrm{eV})$. This is consistent with the stability of the ferrimangetic state. Second, the FM state has an indirect band gap with the $\mathrm{HO}$ and $\mathrm{LU}$ levels located at $\mathrm{K}$ and $\Gamma$, respectively. In the ferrimangetic state, however, the LU level has the highest energy at $\Gamma$ and the band dispersions of the HO and LU levels are almost flat from M to K. This difference comes from the orbital interaction between the spin down $\left(d_{x^{2}-y^{2}}, d_{x y}\right)$ levels of the spin up $\mathrm{Fe}^{3+}$ and $\mathrm{Fe}^{2+}$ ions.

To probe the presence of spin-charge coupling in $\mathrm{LuFe}_{2} \mathrm{O}_{4}$, it is necessary to consider the spin ordering in a $\mathrm{CO}$ state other than the $\sqrt{3} \times \sqrt{3} \mathrm{CO}$ state. The previous electrostatic calculations [5, 18] showed that the chain $\mathrm{CO}$, in which one-dimensional (1D) chains of $\mathrm{Fe}^{2+}$ ions alternate with $1 \mathrm{D}$ chains of $\mathrm{Fe}^{3+}$ ions in each T-sheet [Fig. 2(b)], is only slightly less stable than the $\sqrt{3} \times \sqrt{3} \mathrm{CO}$, and has no FE polarization. We extract exchange parameters by mapping analysis as described above. It is found that the intra-sheet SE between the $\mathrm{Fe}^{3+}$ ions is the strongest $(J=6.7 \mathrm{meV})$ as in the $\sqrt{3} \times \sqrt{3} \mathrm{CO}$ case. All intra-sheet SE's are AFM with $J\left(\mathrm{Fe}^{3+}-\mathrm{Fe}^{3+}\right)>J\left(\mathrm{Fe}^{2+}-\mathrm{Fe}^{3+}\right)>J\left(\mathrm{Fe}^{2+}-\mathrm{Fe}^{2+}\right)$. The inter-sheet $\mathrm{SE}$ between the $\mathrm{Fe}^{3+}$ ions is very weak $(|J|<0.3 \mathrm{meV})$, and that between the $\mathrm{Fe}^{2+}$ and $\mathrm{Fe}^{3+}$ ions is $\mathrm{FM}$ with $J=-1.4 \mathrm{meV}$. Interestingly, the inter-sheet $\mathrm{SE}$ between the $\mathrm{Fe}^{2+}$ ions is rather strongly $\operatorname{AFM}(J=6.3 \mathrm{meV})$. Monte Carlo simulations using these spin exchange parameters indicate that the spin state shown in Fig. 2(b) is the spin ground state. In this spin ordering, the spins within each chain of $\mathrm{Fe}^{2+}$ ions or $\mathrm{Fe}^{3+}$ ions are antiferromagnetically coupled. The NN chains of $\mathrm{Fe}^{2+}$ ions belonging to different T-sheets are coupled antiferromagnetically, whereas the corresponding chains of $\mathrm{Fe}^{3+}$ are almost decoupled.

The above results show that the spin ordering of the chain CO state is dramatically different from that of the $\sqrt{3} \times \sqrt{3} \mathrm{CO}$ state. The most important difference is that the total spin moments are $2.33 \mu_{B} / \mathrm{FU}$ for the $\sqrt{3} \times \sqrt{3} \mathrm{CO}$, but $0 \mu_{B} / \mathrm{FU}$ for the chain CO. This evidences a strong spin-charge coupling in $\mathrm{LuFe}_{2} \mathrm{O}_{4}$. The external magnetic field will have different effects on the two CO states due to the the Zeeman effect. It is expected that the magnetic field will further stabilize the ferrimagnetic $\sqrt{3} \times \sqrt{3}$ CO state. Consequently, an external magnetic field will reduce the extent of charge fluctuation and hence decrease the dielectric constant. This supports our explanation for the giant magnetocapacitance effect of $\mathrm{LuFe}_{2} \mathrm{O}_{4}$ at room temperature [5] .

Without considering the inter-sheet interactions, Naka et al. [18] suggested that the degeneracy of the spin ground state of the $\sqrt{3} \times \sqrt{3} \mathrm{CO}$ state is of the order $\mathrm{O}\left(2^{N / 3}\right)(\mathrm{N}$ is 
the number of the spin sites), which is much larger than the spin degeneracy $\left[\mathrm{O}\left(2^{\sqrt{N}}\right)\right]$ of the chain CO state. Thus, they proposed that spin frustration induces reinforcement of the polar $\sqrt{3} \times \sqrt{3} \mathrm{CO}$ by a gain of spin entropy. However, our calculations show that there are substantial inter-sheet spin exchange interactions between the 2B1 and 3A1 ions, which would remove the macroscopic degeneracy of the spin ground state of the $\sqrt{3} \times \sqrt{3}$ CO state. The macroscopic degeneracy still persists for the chain CO state. Thus, our work provides a picture opposite to what Naka et al. proposed. Furthermore, we find that the $\sqrt{3} \times \sqrt{3}$ $\mathrm{CO}$ state is more favorable for the spin ordering than is the chain CO state; with respect to the paramagnetic state, the spin ground state is lower in energy by $-78 \mathrm{meV} / \mathrm{FU}$ for the $\sqrt{3} \times \sqrt{3} \mathrm{CO}$, but by $-57 \mathrm{meV} / \mathrm{FU}$ for the chain CO. The model of Naka et al. [18] predicts that the polar $\sqrt{3} \times \sqrt{3}$ CO state is destabilized and the electric polarization is reduced by the magnetic field, since it will lift the macroscopic spin degeneracy. In contrast, our work predicts that the magnetic field stabilizes the ferrimagnetic $\sqrt{3} \times \sqrt{3} \mathrm{CO}$ state due to the Zeeman effect, and provides an explanation for why the electric polarization increases when the temperature is lowered below the Neel temperature [3], because the charge fluctuation has an onset well below $T_{C O}[8]$.

In summary, our first principles results explain the experimentally observed Ising ferrimagnetism, and manifest the spin-charge coupling and magnetoelectric effect in $\mathrm{LuFe}_{2} \mathrm{O}_{4}$.

Work at NREL was supported by the U.S. Department of Energy, under Contract No. DE-AC36-08GO28308, and work at NCSU by the U. S. Department of Energy, under Grant DE-FG02-86ER45259. 
[1] T. Kimura, T. Goto, H. Shintani, K. Ishizaka, T. Arima, and Y. Tokura, Nature (London) 426, 55 (2003).

[2] N. Hur, S. Park, P. A. Sharma, J. S. Ahn, S. Guha, and S-W. Cheong, Nature (London) 429, $392(2004)$.

[3] N. Ikeda, H. Ohsumi, K. Ohwada, K. Ishii, T. Inami, K. Kakurai, Y. Murakami, K. Yoshii, S. Mori, Y. Horibe, and H. Kitô, Nature (London) 436, 1136 (2005).

[4] M. A. Subramanian, T. He, J. Chen, N. S. Rogado, T. G. Calvarese, and A. W. Sleight, Adv. Mater. 18, 1737 (2006).

[5] H. J. Xiang and M.-H. Whangbo, Phys. Rev. Lett. 98, 246403 (2007).

[6] Y. Zhang, H. X. Yang, C. Ma, H. F. Tian, and J. Q. Li, Phys. Rev. Lett. 98, 247602 (2007).

[7] M. Angst, R. P. Hermann, A. D. Christianson, M. D. Lumsden, C. Lee, M.-H. Whangbo, J.-W. Kim, P. J. Ryan, S. E. Nagler, W. Tian, R. Jin, B. C. Sales, and D. Mandrus, Phys. Rev. Lett. 101, 227601 (2008).

[8] X. S. Xu, M. Angst, T. V. Brinzari, R. P. Hermann, J. L. Musfeldt, A. D. Christianson, D. Mandrus, B. C. Sales, S. McGill, J.-W. Kim, and Z. Islam, Phys. Rev. Lett. 101, 227602 (2008).

[9] H. J. Xiang and M.-H. Whangbo, Phys. Rev. Lett. 99, 257203 (2007).

[10] H. J. Xiang, S.-H. Wei, M.-H. Whangbo, and J. L. F. Da Silva, Phys. Rev. Lett. 101, 037209 (2008).

[11] J. Iida, M. Tanaka, Y. Nakagawa, S. Funahashi, N. Kimizuka, and S. Takekawa, J. Phys. Soc. Jpn. 62, 1723 (1993).

[12] W. Wu, V. Kiryukhin, H.-J. Noh, K.-T. Ko, J.-H. Park, W. Ratcliff II, P. A. Sharma, N. Harrison, Y. J. Choi, Y. Horibe, S. Lee, S. Park, H. T. Yi, C. L. Zhang, and S.-W. Cheong, Phys. Rev. Lett. 101, 137203 (2008).

[13] D. Dai and M.-H. Whangbo, Inorg. Chem. 44, 4407 (2005).

[14] M. Tanaka, H. Iwasaki, K. Siratori, and I. Shindo, J. Phys. Soc. Jpn. 58, 1433 (1989).

[15] K. Siratori, S. Funahashi, J. Iida, and M. Tanaka, Proc. 6th Intern. Conf. Ferrites, Tokyo and Kyoto, Japan, 1992, p. 703.

[16] A. D. Christianson, M. D. Lumsden, M. Angst, Z. Yamani, W. Tian, R. Jin, E. A. Payzant, 
S. E. Nagler, B. C. Sales, and D. Mandrus, Phys. Rev. Lett. 100, 107601 (2008).

[17] S. Funahashi, J. Akimitsu, K. Siratori, N. Kimizuka, M. Tanaka, and H. Fujishita, J. Phys. Soc. Jpn. 53, 2688 (1984).

[18] M. Naka, A. Nagano, and S. Ishihara, Phys. Rev. B 77, 224441 (2008); A. Nagano, M. Naka, J. Nasu, and S. Ishihara, Phys. Rev. Lett. 99, 217202 (2007).

[19] J. Y. Park, J. H. Park, Y. K. Jeong, and H. M. Jang, Appl. Phys. Lett. 91, 152903 (2007).

[20] P. E. Blöchl, Phys. Rev. B 50, 17953 (1994); G. Kresse and D. Joubert, ibid 59, 1758 (1999).

[21] G. Kresse and J. Furthmüller, Comput. Mater. Sci. 6, 15 (1996); Phys. Rev. B 54, 11169 (1996).

[22] J. P. Perdew, K. Burke, and M. Ernzerhof, Phys. Rev. Lett. 77, 3865 (1996).

[23] A. I. Liechtenstein, V. I. Anisimov and J. Zaanen, Phys. Rev. B 52, R5467 (1995); S. L. Dudarev, G. A. Botton, S. Y. Savrasov, C. J. Humphreys and A. P. Sutton, Phys. Rev. B 57, 1505 (1998).

[24] M.-H. Whangbo, H.-J. Koo and D. Dai, J. Solid State Chem. 176, 417 (2003). 
TABLE I: Calculated superexchange parameters (in meV) in the $\sqrt{3} \times \sqrt{3} \mathrm{CO}$ state of $\mathrm{LuFe}_{2} \mathrm{O}_{4}$ (For the spin sites of the 2A, 3A, 2B and 3B ions,see Fig. 1)

\begin{tabular}{|c|c|c|c|c|}
\hline A-A $J_{3 A 1,2 A 1}$ & $J_{3 A 1,2 A 2}$ & $J_{3 A 1,2 A 3}$ & $J_{2 A 1,2 A 2}$ & $J_{2 A 1,2 A 4}$ \\
\hline 3.2 & 4.0 & 4.7 & 1.9 & 3.6 \\
\hline B-B $J_{3 B 1,3 B 2}$ & $J_{3 B 1,3 B 4}$ & $J_{2 B 1,3 B 1}$ & $J_{2 B 1,3 B 2}$ & $J_{2 B 1,3 B 3}$ \\
\hline 7.0 & 7.6 & 1.5 & 2.8 & 1.3 \\
\hline A-B $J_{3 A 1,3 B 1}$ & $J_{3 A 1,2 B 1}$ & $J_{2 A 1,2 B 1}$ & $J_{2 A 1,3 B 2}$ & $J_{2 A 1,3 B 3}$ \\
\hline 2.0 & 1.9 & $\sim 0$ & -0.6 & 1.2 \\
\hline
\end{tabular}




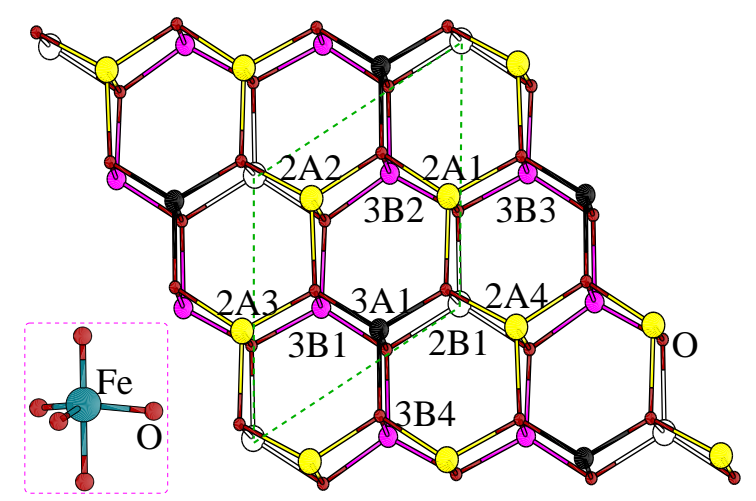

FIG. 1: (Color online) Schematic representation of the $\sqrt{3} \times \sqrt{3}$ CO structure. Large, medium, and small circles represent the $\mathrm{Fe}^{2+}, \mathrm{Fe}^{3+}$, and $\mathrm{O}^{2-}$ ions, respectively. The type A (type B) T-sheet has the honeycomb network of $\mathrm{Fe}^{2+}\left(\mathrm{Fe}^{3+}\right)$ ions with a $\mathrm{Fe}^{3+}\left(\mathrm{Fe}^{2+}\right)$ ion at the center of each hexagon. $2 \mathrm{~A}$ and $3 \mathrm{~A}(2 \mathrm{~B}$ and $3 \mathrm{~B})$ refer to the $\mathrm{Fe}^{2+}$ and $\mathrm{Fe}^{3+}$ ions of the type $\mathrm{A}$ (type $\mathrm{B}$ ) T-sheet, respetively. The region enclosed by dashed lines indicates the unit cell of the CO structure. There is a mirror plane of symmetry, which is parallel to the $c$ axis and crosses the $3 \mathrm{~A} 1$ and $2 \mathrm{~B} 1$ sites. The inset shows an isolated $\mathrm{FeO}_{5}$ trigonal bipyramid.

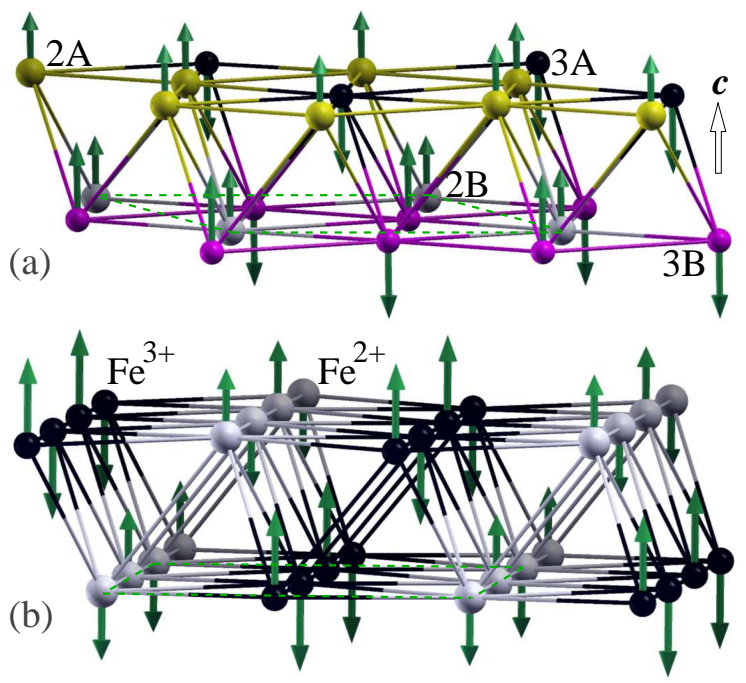

FIG. 2: (Color online) Schematic representations of (a) the spin ground state of the $\sqrt{3} \times \sqrt{3}$ $\mathrm{CO}$ structure and (b) one of the macroscopic spin ground states of the chain CO structure. The arrows denote the spin directions. The region enclosed by the dashed lines on the bottom T-sheet indicates the magnetic unit cell of the spin structure. 


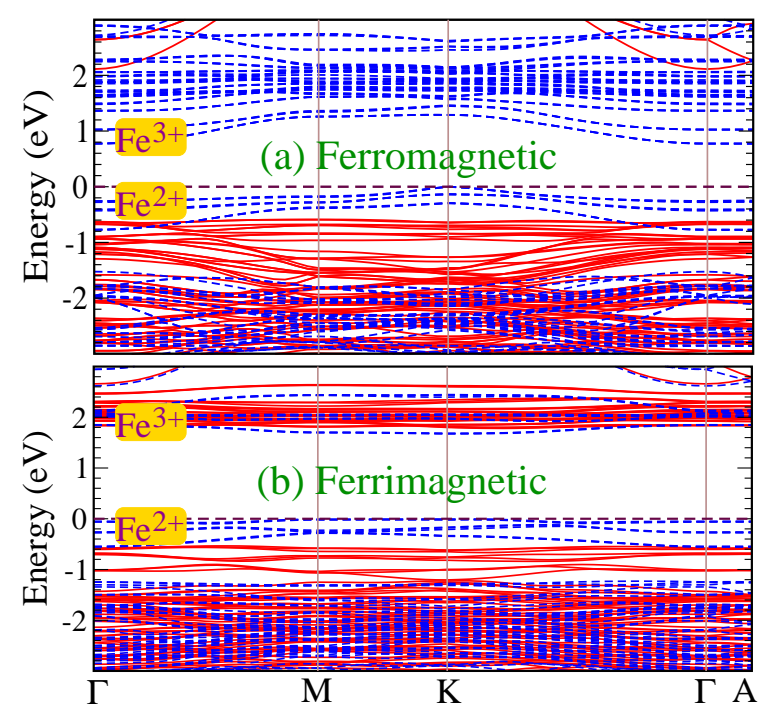

FIG. 3: (Color online) Band structures calculated for (a) the FM state and (b) the ferrimagnetic state of the $\sqrt{3} \times \sqrt{3} \mathrm{CO}$ structure of $\mathrm{LuFe}_{2} \mathrm{O}_{4}$. The solid and dashed lines represent the up-spin and down-spin bands, respectively. The $\sqrt{3} \times \sqrt{3} \times 1$ hexagonal cell is used in the calculations. 